\title{
Photo Quiz; Anthrax or Orf?
}

\author{
Necla Tülek², Esra Kaya Kılıç², GünayTuncer Ertem², Esra Yüksekkaya² \\ 1 Department of Basic Medical Medical Sciences, Atılım University School of Medicine, Ankara, Turkey; \\ 2 Department of Infectious Diseases and Clinical Microbiology, Ankara Training and Research Hospital, Ankara, Turkey
} 38-year-old man presented to the hospital with complaints of fever and multiple lesions on his face, hands, and arms. The lesions were appeared five days earlier before admission as painless small papules and developed into larger lesions with the darkish center. He was experiencing a fever for the last 24 hours. He was a farmer, he sacrificed and butchered a bull at the Eid al-Adha, ten days ago. He reported that he was healthy previously and did not use any drug. On physical examination, his body temperature was $39^{\circ} \mathrm{C}$. He had multiple lesions on arms, hands, face and erythematous diffuse edema on his right hand, on $4^{\text {th }}$ finger extending to the arm. There were some satellite lesions on the left arm (Figure1, 2), as well. He had a red lymphangitic streak on the right arm that extended from the hands toward the axillae with a palpable and tender axillary lymph node $2 \mathrm{~cm}$ in diameter. Laboratory examination revealed white blood count 11600 cells/ $\mathrm{LL}$ with left shift, and C-reactive protein $85 \mathrm{mg} /$ $d L\left(N\right.$ : 0-5). Aspiration material was obtained from the lesion on the $4^{\text {th }}$ finger, stained with Gram stain and cultured.

Which is your presumptive diagnosis? Orf or Anthrax?

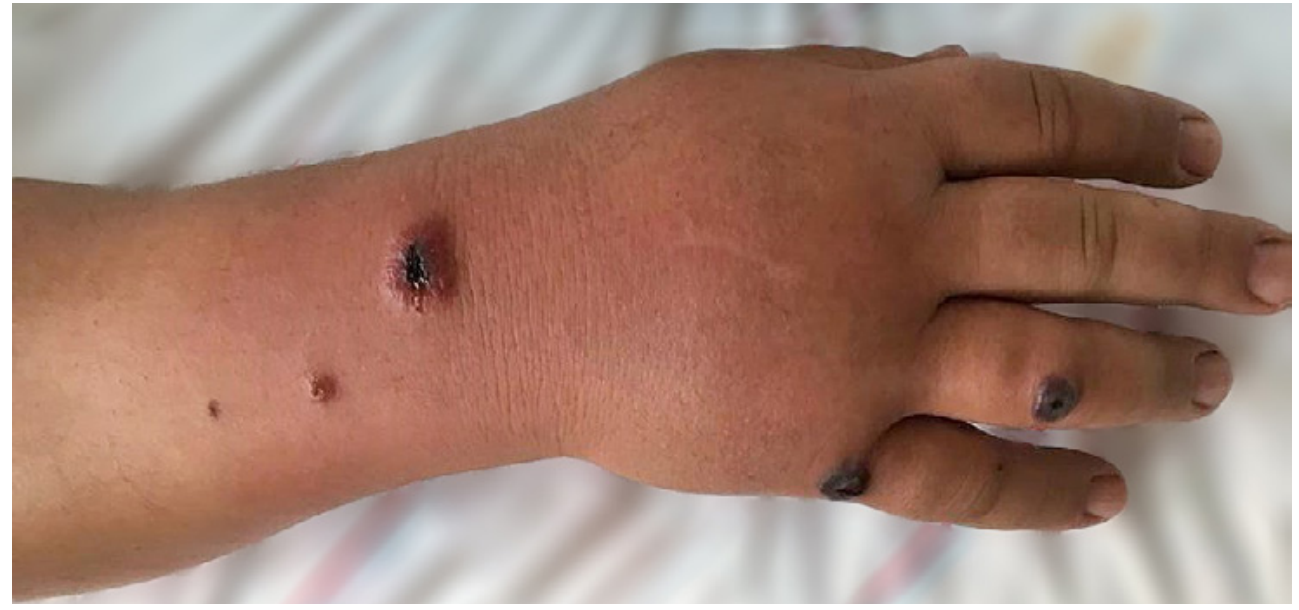

Figure 1. Multiple dark papules with central umbilicus on patient's right hand and on the $4^{\text {th }}$ and the $5^{\text {th }}$ fingers including diffuse edema.

\author{
Corresponding Author: \\ Necla Tülek \\ E-mail: \\ ntulek@gmail.com
}

Received: February 19, 2019 Accepted: March 27, 2019 Published: May 30, 2019

Suggested citation: Tülek N, Kılıç Kaya E, Tuncer Ertem G, Yüksekkaya E. Photo Quiz; Anthrax or Orf? Infect Dis Clin Microbiol 2019; 1: 55-58.

DOI: $10.5152 /$ idcm.2019.19002 


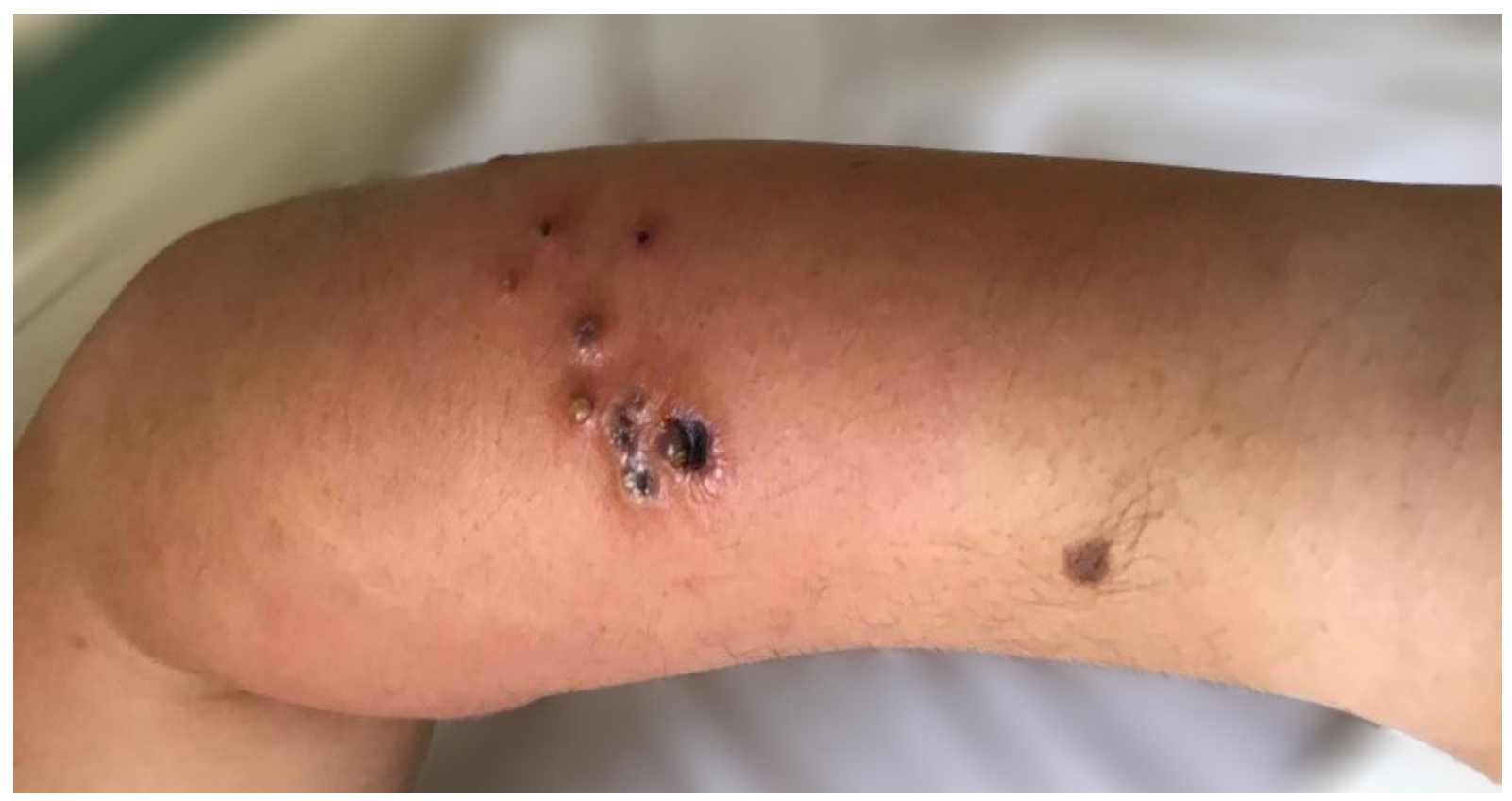

Figure 2. Similar lesions as in Figure 1 on extensor surface of the left arm.

\section{DISCUSSION}

\section{Diagnosis: Anthrax}

Gram-positive large bacilli were detected on Gram stain of fluid material obtained from underneath of eschar by needle aspiration and Bacillus anthracis grew on blood agar.

Both of orf and anthrax are zoonotic diseases.

\section{ORF}

Orf, also known as ecthyma contagiosum, is caused by the epitheliotropic parapoxvirus which is a member of Poxviridae. Orf is most often transmitted to humans through direct contact inoculation with infected animals such as sheep, goats or their fomites. Breakdown of skin integrity is a risk factor for transmission. Those at highest risk for infection are shepherds, veterinarians, butchers, and farmers. It can be transmitted through handling the meat, wool, carcasses even during the household meat preparation (1).

The incubation period is 2-7 days. Lesions are localized to the epithelium at the site of inoculation, frequently develop on the extensor surface of the hands, digits, and autoinoculation to the other body surfaces may occur. A single or multiple lesions may develop. The initial lesions are maculopapular or target site with red-blue color then progress to hemorrhagic pustules or bulla containing central crust and bleed easily. After that, a red weeping nodule with ulceration and finally a thin, yellowish crust develops. Lesions resolve spontaneously in immunocompetent patients, complete healing without scarring may take 2-6 weeks. A mild fever and lymphadenopathy may be seen in the patients. secondary bacterial infections may also occur (2).

The diagnosis of the disease in humans is based on the epidemiological history and characteristic features of the skin lesions. The differential diagnosis includes cutaneous anthrax, pyoderma gangrenosum, tularemia, herpetic whitlow, milker's nodule, and Mycobacterium marinum granulomas. Real timePCR is a more sensitive method for the diagnosis. Serology, electron microscopic examination and, viral culture are additional diagnostic methods. (3).

The disease is self-limited and the main therapy is the wound care. There is no specific treatment for 
orf but cidofovir, cryotherapy, and surgical therapy have been recommended as therapeutic alternatives in immunocompromised patients.

For the prevention of disease, basic hygienic principles including the use of barrier measures such as gloves when handling meats and hand hygiene must be applied. Control of the disease in animals is essential (4).

\section{CUTANEOUS ANTHRAX}

Cutaneous anthrax is the most common form of the naturally occurring disease. Exposure to Bacillus anthracis may occur through agricultural or industrial contact with infected animals or animal products. Risk groups are similar to orf; shepherds, farmers, veterinarians, workers in animal production facilities. Human-to-human transmission is not expected (5).

The primary lesion develops in within 5-7 days (range 1-12 days) after penetration of Bacillus anthracis spores through skin lacerations, cuts, abrasions, or insect bites. Spores vegetate and bacilli multiply at the site of the entry. Cutaneous anthrax most commonly affects the upper extremities, hands, necks, and face. Hematogenous dissemination occurs in $5-10 \%$ of untreated cases. Skin lesions of orf and anthrax exhibit a similar appearance initially but initial anthrax lesion is a painless, pruritic macule or papule then turns into a vesicle and bulla in 1-2 cm diameter quickly in a few days. The bulla ruptures and is followed by the formation of a necrotic ulcer with a black depressed central eschar and a round raised edge within 7-10 days. Healing usually results in scar formation in contradistinction contrast to Orf. A major diagnostic feature of the cutaneous anthrax is the development of non-pitting edema around the lesion due to toxin release. Lymphangitis, regional painful lymphadenopathy may occur and systemic symptoms, including fever, malaise can accompany the cutaneous lesion (6).

The presumptive diagnosis of cutaneous anthrax is based on the epidemiological history and characteristic features of the skin lesions. Bacillus anthracis can be identified by direct Gram or Methylene
Blue staining of the specimens obtained from vesicular fluid or the base of the lesion. The presence of gram-positive rods and few polymorphonuclear leukocytes are strongly suggestive of cutaneous anthrax. Bacillus anthracis grows easily on standard culture media but biosafety level II precautions are needed. Nonculture based laboratory tests are PCR assay, immunohistochemical staining of tissues, and detection of anti-protective antigen immunoglobulin IgG by an enzyme-linked immunosorbent assay (7).

Patients with cutaneous anthrax without systemic involvement should receive oral antimicrobial therapy. The preferred agent used to treat naturally acquired anthrax is penicillin. Ciprofloxacin, levofloxacin, moxifloxacin, doxycycline or clindamycin are other effective agents. Treatment duration is 7-10 days. Control of the disease in animals is essential for the prevention in humans (8).

Major differences between the lesions of anthrax and orf diseases:

- Both of the lesions start as a papule but the orf lesion is smaller and red-blue color.

- Anthrax lesion forms a vesicle in 24-48 hours and becomes an ulcer with a surrounding edematous halo whereas orf lesion takes an iris-like shape with a central red nodule surrounded by a white circle and a red periphery.

- Anthrax lesions may become edematous and necrotic but are usually not purulent. Orf lesions are weeping nodules with an exudative appearance.

- A black ulcer is characteristic for anthrax and develops within 7-10 days and lasts 7-14 days whereas orf lesions heal after the development of thick dry crust in 5-6 weeks. 


\section{REFERENCES}

1 Petersen BW. Other poxviruses that infect humans. In: Damon IK, ed. Mandell, Douglas, and Bennett's Principles and Practice of Infectious Diseases. Philadelphia, PA: Elsevier, 2015: 1703-6.

2 Abbas O. Orf virus infection. UpToDate (www.uptodate.com). Date last accessed: Feb 17, 2019.

3 Caravaglio JV, Khachemoune A. Orf virus infection in humans: a review with a focus on advances in diagnosis and treatment. J Drugs Dermatol. 2017; 16: 684-9.

4 Bala JA, Balakrishnan KN, Abdullah AA, et al. The re-emerging of orf virus infection: A call for surveillance, vaccination and effective control measures. Microb Pathog. 2018; 120: 55-63.

5 Martin GJ, Friedlander AM. Bacillus anthracis (Anthrax). In: Mandell GL, Bennett JE, Dolin R, eds. Mandell, Douglas, and Ben- nett's Principles and Practice of Infectious Diseases, Updated Edition. 8th ed. Philadelphia, PA: Elsevier Saunders, 2015: 2391e2-2409e2.

6 Doganay M, Metan G, Alp E. A review of cutaneous anthrax and its outcome. J Infect Public Health. 2010; 3: 98-105.

7 Wilson KH. Clinical manifestations and diagnosis of anthrax. UpToDate (www.uptodate.com). Date last accessed: Feb 17, 2019.

8 Hendricks KA, Wright ME, Shadomy SV, et al. Centers for disease control and prevention expert panel meetings on prevention and treatment of anthrax in adults. Emerg Infect Dis. 2014; 20: e130687. 